ESAIM: PROCEEDINGS, August 2009, Vol. 28, p. 182-194

M. Ismail, B. Maury \& J.-F. Gerbeau, Editors

\title{
DYNAMICS OF RED BLOOD CELLS IN 2D
}

\author{
Cuc Bui ${ }^{1}$, Vanessa Lleras ${ }^{2}$ And Olivier Pantz ${ }^{3}$
}

\begin{abstract}
Blood is essentially composed of red blood cells, white blood cells and platelets suspended in a fluid (blood plasma). If it can be considered as a homogeneous fluid when circulating in vessels of large diameter, this approximation is no longer valid when it reaches vessels with diameter of an order of magnitude comparable to that of the cells it carries. In this case, the influence of the cells on the flow can no longer be homogenized. Therefore, the mechanical behavior of red blood cells (which account for $99 \%$ of the cells presenting in the blood), their interaction with the surrounding fluid or between themselves (by contact) must be taken into account. Numerical tool plays thus an essential role: it enables to validate the advanced physical models, to access to data difficult to obtain experimentally and to determine the dependence of the flow behavior on the parameters of the model. In this article, we propose a numerical method which allows to take into account these three essential aspects (mechanical behavior of red blood cells, fluid/structures interactions and structures/structures contact interactions). Our study is limited to the two-dimensional case which, although simplistic, allows us to reproduce a quite large range of experimental observations as shown in the numerical simulations obtained.
\end{abstract}

Résumé. Le sang est pour l'essentiel composé de globules rouges, blancs et de plaquettes en suspension dans un liquide (le plasma sanguin). S'il peut être considéré comme un fluide homogène lorsqu'il circule dans des vaisseaux de diamètre important, cette approximation n'est plus valable dès qu'il atteint des vaisseaux dont la taille est d'un ordre de grandeur comparable aux cellules qu'il transporte. Dans ce cas, l'influence de ces dernières sur l'écoulement ne peut plus être homogénéisée ("moyennée"). Le comportement mécanique des globules rouges (qui comptent pour $99 \%$ des cellules présentent dans le sang), leur interaction avec le fluide environnant ou entre eux même (par contact) doivent être pris en compte. L'outil numérique joue alors un rôle primordial: il permet de valider les modèles physiques avancés, d'accéder à des données difficiles à obtenir expérimentalement et de déterminer la dépendance du comportement de l'écoulement en fonctions des paramètres du modèle. Dans cette note, nous proposons une méthode numérique permettant de prendre en compte ces trois aspects essentiels (comportement mécanique des globules rouges, interactions fluide/structures et interactions de contacts structures/structures). Nous limitons notre étude au cas bidimensionnel qui, bien que simplificateur, nous permet de reproduire une palette assez importante d'observations expérimentales comme l'illustre les simulations numériques obtenues.

\footnotetext{
${ }^{1}$ Laboratoire J.L. Lions, UPMC Univ Paris 06, UMR 7598, 75005 Paris, France

${ }^{2}$ Laboratoire de Mathématiques, Université de Franche Comté, CNRS UMR 6623, 16 route de Gray, 25030 Besançon cedex, France

${ }^{3}$ CMAP, École Polytechnique, 91128 Palaiseau Cedex, France
}

(C) EDP Sciences, SMAI 2009 


\section{INTRODUCTION}

If the blood could be considered as a homogeneous fluid in large domains, such an approximation is meaningless in small vessels of same characteristic scale than the cells it convects. Those are essentially (for $99 \%$ of the volume) Red Blood Cells (RBCs) plus some white blood cells and platelets. Almost half of the blood is composed of RBCs, thus they are playing a key role in the blood rheology. This role depends on the mechanical behavior of the RBCs, on the interactions between their membrane and the surrounding fluid, and possible interactions between the RBCs themselves. In this context, numerical simulations could be a useful tool as they enable to test different modelings, to perform "numerical" experiments maybe difficult to realize in "vitro" or in "vivo", to determine different physical parameters by comparing numerical and experimental results, to study the effects of a modification of those parameters, which are, in vivo, possible sources of diseases. This has stimulated an intense activity in recent years and a large range of numerical methods has been proposed, most of them in bi-dimensional context: Immersed Boundary [17], Penality [16], Lattice Boltzmann [20,28], Multiparticle collision Dynamics [21,22], Integral Method [15,24,25], Level Set and phase field [3,4,8], Finite Element conformal method [6]. In parallel, many experiments have been performed, enabling a better understanding of vesicles' dynamics and bringing to light different kinds of phenomenon: lift (vesicles tend to concentrate in the middle of the vessels, pushed by the hydrodynamic forces induced by the walls of the vessels $[1,5]$ ), various behavior of the vesicles in a shear flow (tumbling, tank-treading or vacillating breathing $[13,14,18,19]$ ), formation of rouleaux (aggregation of vesicles), parachute shape of the vesicles under Poiseuille flow [27]. Finally, several theoretical studies have focused on the analytical resolution of simplified versions of the equations governing vesicles dynamics in particular flows.

In this article, we present a numerical method in order to solve the fluid/structure system formed by the RBCs and the surrounding fluid in a bi-dimensional setting. The RBCs' membrane energy is assumed to be proportional to the square of its curvature and the surrounding fluid to be incompressible and Newtonian $[7,11]$. A finite element method with conformal meshes for both the fluid and the membranes has been implemented with FreeFem $++^{1}$, a free finite element software [10]. Several problems have to be taken up. The first problem consists in taking correctly into account the fluid/structure coupling so to obtain a stable scheme. Due to the incompressibility of the membrane, the surface of the $\mathrm{RBC}$ remains constant in time. Moreover, as the fluid is incompressible and the membrane assumed to be impermeable, the volume of the vesicle remains constant as well [26]. Both of these constraints have to be verified with enough accuracy to obtain realistic simulations. Finally, a robust algorithm has to be able to deal with the eventual formation of contacts between RBCs or between RBCs and the vessels' walls. If both RBCs and the walls of the vessels are regular and a non slipping condition is assumed for the fluid at the boundary of its domain, hydrodynamic forces prevent any contact in finite time [12]. Nevertheless, it seems that adhesive intercellular interactions (not taken into account in the present article) competing with hydrodynamic forces could lead to contacts between cells. Anyway, contacts could always occur "numerically" because of the discretization of the problem and the important concentration of RBCs. In order to prevent overlapping of the RBCs, we add a new constraint so that the distance between two RBCs or between the RBCs and the walls remains great than a small threshold [23].

Our article is organized in three parts. In Section 2, we present the modeling used in our simulation describing the RBC dynamics. Section 3 is devoted to the conformal method implemented, whereas Section 4 presents several numerical results obtained. Finally, in the last section, some conclusions are drawn and future possible work directions are given.

\section{Modelling of RED BLOOD CELlS in A FLOW}

\subsection{Mechanical behavior of RBCs}

The mechanical behavior of a RBC is driven by the nature of its membrane which is mainly made of a lipid bilayer. Lipid bilayers are self-assembled structures of phospholipids which are small molecules containing a

\footnotetext{
${ }^{1}$ http://www.freefem.org/ff++/
} 
negatively charged phosphate group (called the head) and two highly hydrophobic fatty acid chains (called the tails). In an aqueous environment, phospholipids spontaneously form a double layer whose configuration enables to isolate the tails of the phospholipids from the watery environment. Modifying the area of a such lipid bilayer is very energy costly as it will expose some of the tails to the environment. Thus, their surface (that is the perimeter in the $2 \mathrm{~d}$ case as considered here) remains constant. If lipid bilayers set almost no resistance to shear stress, they do resist to bending. A widely use model is to consider that lipid bilayer could be endowed with an elastic energy, known as the Helfrich functional (sometimes named after Willmore in other contexts)

$$
J(\varphi)=\frac{1}{2} \int_{\Sigma} k|H|^{2} d s
$$

where $\Sigma$ is the surface occupied by the lipid bilayer, $\varphi$ is the deformation of the RBC, $d s$ the surface measure, $H$ the mean curvature of the layer and $k$ the bending elastic modulus.

Note that beside the lipid bilayer, RBC are also composed of a protein skeleton. This skeleton ensures a small resistance of the RBCs to shear stress. The total energy of a RBC is given by

$$
J_{R B C}(\varphi)=\frac{1}{2} \int_{\Sigma} k|H|^{2} d s+\frac{1}{2} \int_{\Sigma_{0}} W(\nabla \varphi) d s,
$$

where $\Sigma_{0}$ is the reference configuration of the membrane and $W$ the membrane stored-energy function. Moreover the membrane stored-energy $W(\nabla \varphi)$ only depends on the metric tensor $\nabla \varphi^{T} \nabla \varphi$. In a two-dimensonal framework, the local inextensibility condition implies the metric tensor to be equal to the identity. It follows that $J_{R B C}=J$ (up to a constant). The situation is quite different in a three-dimensional setting, where the local conservation of the area of the membrane does not imply that the metric tensor of the deformation is reduced to the identity. As we work in a two-dimensinal setting, the distinction between RBCs and vesicles, whose internal energies are respecticely given by (1) and (2) is irrelevant.

\subsection{Surrounding Fluid}

We assume that the RBCs are surrounded by an incompressible Newtonian fluid characterized by its density $\rho$ and its viscosity $\mu$. We use Navier-Stokes equation, and, if the inertia of the fluid can be neglected, Stokes equation instead.

\subsection{Variational formulation}

We denote by $\Omega$ the domain of the fluid. The deformations of the RBCs are denoted by $\varphi: \Sigma_{0} \rightarrow \bar{\Omega}$, where $\Sigma_{0}$ is their reference configuration. The viscosity of the fluid $\mu$ is assumed to be constant inside as well as outside the vesicles respectively equal to $\mu_{\text {in }}$ and $\mu_{\text {out }}$. The inertia of the membranes is always neglected. The problem consists in determining the fluid velocity $u:] 0, T\left[\times \Omega \rightarrow \mathbb{R}^{2}\right.$, the deformations of the vesicles $\left.\varphi:\right] 0, T\left[\times \Sigma_{0} \rightarrow \mathbb{R}^{2}\right.$, the pressure $p:] 0, T\left[\times \Omega \rightarrow \mathbb{R}\right.$ and the inextensional pressure $\left.p_{\text {inext }}:\right] 0, T\left[\times \Sigma_{0} \rightarrow \mathbb{R}\right.$, such that for all test functions $v, q$ and $q_{\text {inext }}$, we have

$$
\begin{gathered}
\int_{\Omega} \rho \frac{\partial u}{\partial t} v+\rho(u \cdot \nabla u) v-(\nabla \cdot v) p+2 \mu e(u) \cdot e(v) d x+\left\langle J^{\prime}(\varphi), v \circ \varphi\right\rangle+\int_{\Sigma}\left(\tau \cdot \frac{\partial v}{\partial \tau}\right) p_{\text {inext }} \circ \varphi^{-1} d s=0, \\
\int_{\Sigma}\left(\tau \cdot \frac{\partial u}{\partial \tau}\right) q_{\text {inext }} \circ \varphi^{-1} d s=0, \\
\int_{\Omega}(\nabla \cdot u) q d x=0, \\
\frac{\partial \varphi}{\partial t}=u \circ \varphi
\end{gathered}
$$


where $\rho$ is the density of the fluid and $e(u)=\left(\nabla u+\nabla^{T} u\right) / 2$ is the strain rate. Boundary conditions have to be added to close the system. The reference configuration $\Sigma_{0}$ of the membrane of the RBCs is a union of loops diffeomorphic to the unit circle $S^{1}$ and $\Sigma=\varphi\left(\Sigma_{0}\right)$.

The classical Navier-Stokes equation (3) is supplemented with two additional terms due to the action of the membrane on the fluid. As the inertia of the membranes of the RBCs is neglected, the forces they exert on the fluid are equal to their internal stress and have two components. One of them follows directly from the bending energy $J$, while the other is due to the membrane incompressibility. The incompressibility constraint of the membrane is imposed through the introduction of the Lagrange multiplier $p_{\text {inext }}$ which we call the "inextensional pressure" as it plays a similar role like the pressure $p$ ensuring the incompressibility of the fluid. The incompressibility of the membrane and of the fluid are respectively imposed through equations (4) and (5), while equation (6) simply expresses that the velocity of the membrane is equal to the velocity of the fluid.

To be more precise, the local inextensibility condition follows both from equations (4) and (6). Deriving (6) with respect to the $s \in \Sigma_{0}$, we obtain that

$$
(\nabla u) \circ \varphi(t, s) \frac{\partial \varphi}{\partial s}(t, s)=\frac{\partial^{2} \varphi}{\partial s \partial t}(t, s) .
$$

As the unitary tangential vector to the surface of the $\mathrm{RBC}$ reads as

$$
\tau \circ \varphi=\left|\frac{\partial \varphi}{\partial s}\right|^{-1} \frac{\partial \varphi}{\partial s}
$$

it follows that

$$
\frac{\partial u}{\partial \tau} \circ \varphi(t, s)\left|\frac{\partial \varphi}{\partial s}\right|=\frac{\partial^{2} \varphi}{\partial s \partial t}(t, s)
$$

and from the equation (4), we obtain

$$
\frac{\partial \varphi}{\partial s} \cdot \frac{\partial^{2} \varphi}{\partial s \partial t}(t, s)=0
$$

and finally the local inextensibility of the membrane

$$
\frac{\partial}{\partial t}\left|\frac{\partial \varphi}{\partial s}\right|^{2}=0 .
$$

\section{NumericAl SCHEME}

\subsection{Time discretization}

We use a finite difference method for the time discretization. Let $\Delta t$ be the time step and $u^{n}, \varphi^{n}, p^{n}$ and $p_{\text {inext }}^{n}$ be the approximations of $u, \varphi, p$ and $p_{\text {inext }}$ at time $t_{n}=n \Delta t$. Our scheme consists in solving the following sequence of problems: Find $u^{n+1}, \varphi^{n+1}, p^{n+1}$ and $p_{\text {inext }}^{n+1}$ such that for all test functions $v, q$ and $q_{\text {inext }}$ :

$$
\begin{gathered}
\int_{\Omega} \rho \frac{u^{n+1}-u^{n} \circ X^{n}}{\Delta t} v-(\nabla \cdot v) p^{n+1}+2 \mu e\left(u^{n+1}\right) \cdot e(v) d x \\
+\left\langle J^{\prime}\left(\varphi^{n+1}\right), v \circ \varphi^{n}\right\rangle+\int_{\Sigma}\left(\tau \cdot \frac{\partial v}{\partial \tau}\right) p_{\text {inext }}^{n+1} \circ\left(\varphi^{n}\right)^{-1} d s=0, \\
\int_{\Omega}\left(\nabla \cdot u^{n+1}\right) q d x=0, \\
\int_{\Sigma}\left(\tau \cdot \frac{\partial u^{n+1}}{\partial \tau}\right) q_{\text {inext }} \circ\left(\varphi^{n}\right)^{-1} d s=0,
\end{gathered}
$$


with

$$
\varphi^{n+1}=\varphi^{n}+\Delta t u^{n+1}
$$

and $\left.X^{n}:\right]-\infty, \infty[\times \Omega \rightarrow \Omega$ is defined by

$$
\begin{cases}X^{n}(0, x)=x & \text { for all } x \in \Omega \\ \frac{\partial X^{n}}{\partial t}(t, x)=u^{n}\left(X^{n}(t, x)\right) & \text { for all }(t, x) \in]-\infty, \infty[\times \Omega\end{cases}
$$

\subsection{Space discretization}

The deformation of the RBCs is discretized using $P 1$-Lagrange finite elements over a mesh $\mathcal{S}_{h}^{0}$ of $\Sigma_{0}$ independent of the time $t_{n}$. Accordingly, the inextensional pressure $p_{\text {inext }}$ is discretized with $P 0$-Lagrange finite elements over the same mesh. We denote by $V_{h}$ and $W_{h}$ the set of $P 1$ and $P 0$-Lagrange finite elements over $\mathcal{S}_{h}^{0}$, that is

$$
\begin{gathered}
V_{h}=\left\{\varphi_{h} \in H^{1}\left(\Sigma_{0} ; \mathbb{R}^{2}\right) \text { such that } \varphi_{\left.h\right|_{e}} \in P_{1} \text { for all edges } e \text { of } \mathcal{S}_{h}^{0}\right\} \\
W_{h}=\left\{p_{h} \in L^{2}\left(\Sigma_{0} ; \mathbb{R}\right) \text { such that } p_{\left.\right|_{e}} \in P_{0} \text { for all edges } e \text { of } \mathcal{S}_{h}^{0}\right\} .
\end{gathered}
$$

On the other hand, we use a conformal mesh to compute the velocity and pressure at each time $t_{n}$. More precisely, for each iteration $n>0$, we introduce the mesh $\mathcal{S}_{h}^{n}=\varphi^{n}\left(\mathcal{S}_{h}^{0}\right)$ of $\Sigma_{h}^{n}=\varphi^{n}\left(\Sigma_{0}\right)$. The velocity and pressure at time $n+1$ are computed on a regular triangular mesh $\mathcal{T}_{h}^{n}$ such that $\mathcal{S}_{h}^{n}$ is a submesh of $\mathcal{T}_{h}^{n}$, that is, each edge of $\mathcal{S}_{h}^{n}$ is an edge of $\mathcal{T}_{h}^{n}$. A $P 1$-bubble/P1 formulation is used, e.g. $P 1$-bubble for the velocity $u$ and $P 1$ finite elements for the pressure $p$. We denote by $P_{h}^{n}$ and $U_{h}^{n}$ the set of $P 1$ and $P 1$-bubble Lagrange finite elements over $\mathcal{T}_{h}^{n}$, that is

$$
P_{h}^{n}=\left\{v_{h} \in H^{1}(\Omega ; \mathbb{R}) \text { such that } v_{\left.h\right|_{K}} \in P_{1} \text { for all triangles } K \text { of } \mathcal{T}_{h}^{n}\right\}
$$

and

$$
U_{h}^{n}=\left(P_{h}^{n}\right)^{2} \oplus B_{h}^{n}
$$

where

$$
B_{h}^{n}=\left\{v_{h} \in H^{1}\left(\Omega ; \mathbb{R}^{2}\right) \text { such that } v_{\left.h\right|_{K}} \in P_{3} \text { for all triangles } K \text { and } v_{\left.h\right|_{e}}=0 \text { for all edges } e \text { of } \mathcal{T}_{h}^{n}\right\} .
$$

\subsection{Approximation of the membrane energy}

As the membrane of the RBCs are assumed to be inextensible, the Helfrich functional (1) takes a particular simple form. Indeed, we have

$$
J(\varphi)=\frac{1}{2} \int_{\Sigma_{0}} k\left|\frac{d^{2} \varphi}{d s^{2}}\right|^{2} d s .
$$

Unfortunately, this energy is never finite for elements of the space discretization chosen for the deformation (that is $P 1$ Lagrange finite elements). In order to overcome this problem, we approximate the functional $J$ by

$$
J_{h}\left(\varphi_{h}\right)=\inf _{\substack{\Phi_{h} \in H^{2}\left(\Sigma_{0} ; \mathbb{R}^{2}\right) \\ \Phi_{h}\left(x_{i}\right)=\varphi_{h}\left(x_{i}\right)}} J\left(\Phi_{h}\right)
$$

where $\left(x_{i}\right)$ spans the set of vertices of the mesh $\mathcal{S}_{h}^{0}$ of $\Sigma_{0}$. The infimum of (8) is achieved for P3 Lagrange finite elements $\Phi_{h}$ called the spline cubic associated to $\varphi$. We set

$$
R_{h}=\left\{\Phi_{h} \in H^{1}\left(\Sigma_{0} ; \mathbb{R}^{2}\right) \text { such that }\left.\Phi_{h}\right|_{e} \in P_{3} \text { for all edges } e \text { of } \mathcal{S}_{h}^{0}\right\},
$$


and we have

$$
\begin{array}{r}
J_{h}\left(\varphi_{h}\right)=\inf \left\{\frac{1}{2} \sum_{e \in \mathcal{E}_{h}^{n}} \int_{e} k\left|\frac{d^{2}\left(\Phi_{h}^{n+1} \circ\left(\varphi^{n}\right)^{-1}\right)}{d s^{2}}\right|^{2} d s: \Phi^{n+1} \in R_{h} ; \Phi\left(x_{i}\right)=\varphi\left(x_{i}\right) \text { for all nodes } x_{i} \in \mathcal{S}_{h}^{0}\right. \\
\text { and } \left.b_{n}\left(\Phi_{h}, q_{\tau}\right)=0 \text { for all } q_{\tau} \in V_{h}\right\},
\end{array}
$$

where

$$
b_{n}\left(\Phi_{h}, q_{\tau}\right)=\sum_{e \in \mathcal{E}_{h}^{n}} \int_{e} \frac{d^{2}\left(\Phi_{h} \circ\left(\varphi^{n}\right)^{-1}\right)}{d s^{2}} \cdot q_{\tau} \circ\left(\varphi^{n}\right)^{-1} d s+\int_{\Sigma} \frac{d\left(\Phi_{h}^{n+1} \circ\left(\varphi^{n}\right)^{-1}\right)}{d s} \cdot \frac{d\left(q_{\tau} \circ\left(\varphi^{n}\right)^{-1}\right)}{d s} d s
$$

The solution of the minimization problem (8) belongs to the set of spline cubic Hermite finite elements of $\mathcal{S}_{h}^{0}$. Unfortunately, the finite elements software used in this article is retricted to Lagrange finite elements. We circumvent this limitation by reformulating this minimization problem as in (9) over the Lagrange finite space $R_{h}$. The additional constraint $b_{n}\left(\Phi_{h}, q_{\tau}\right)=0$ added ensure the admissible solutions $\Phi_{h}$ to belong to $H^{2}\left(\Sigma_{0}\right)$.

In order to solve an approximation of (7) we thus introduce $\Phi_{h} \in R_{h}$ as a new variable in our system. Moreover, two Lagrange multipliers are also introduced: $p_{\tau}$ to ensure that $\Phi_{h}$ belongs to the space $H^{2}$ and $p_{c}$ to ensure the condition $\Phi_{h}\left(x_{i}\right)=\varphi_{h}\left(x_{i}\right)$. Both multipliers are $P 1$ finite elements with values in $\mathbb{R}^{2}$. The equation (7) rewritten using this discrete setting has the following form: Find $\left(u^{n+1}, \Phi_{h}^{n+1}, p^{n+1}, p_{\text {inext }}^{n+1}, p_{\tau}^{n+1}, p_{c}^{n+1}\right) \in$ $U_{h}^{n} \times R_{h} \times P_{h}^{n} \times W_{h} \times V_{h} \times V_{h}$ such that for all test functions $\left(v, \Psi_{h}, q, q_{\text {inext }}, q_{\tau}, q_{c}\right) \in U_{h}^{n} \times R_{h} \times P_{h}^{n} \times W_{h} \times V_{h} \times V_{h}$, we have

$$
\begin{aligned}
& \int_{\Omega} \rho \frac{u^{n+1}-u^{n} \circ X^{n}}{\Delta t} v-(\nabla \cdot v) p^{n+1}+2 \mu e\left(u^{n+1}\right) \cdot e(v) d x-\Delta t \sum_{x \in \mathcal{V}_{h}^{0}} h_{i}^{n} v(x) \cdot p_{c}^{n+1}(x) \\
& +\int_{\Sigma}\left(\tau \cdot \frac{\partial v}{\partial \tau}\right) p_{\text {inext }} \circ\left(\varphi^{n}\right)^{-1} d s=0, \\
& \sum_{e \in \mathcal{E}_{h}^{n}} \int_{e} k \frac{d^{2}\left(\Phi_{h}^{n+1} \circ\left(\varphi^{n}\right)^{-1}\right)}{d s^{2}} \frac{d^{2}\left(\Psi \circ\left(\varphi^{n}\right)^{-1}\right)}{d s^{2}}+\frac{d^{2}\left(\Psi \circ\left(\varphi^{n}\right)^{-1}\right)}{d s^{2}} \cdot p_{\tau}^{n+1} \circ\left(\varphi^{n}\right)^{-1} d s \\
& +\int_{\Sigma} \frac{d\left(\Psi \circ\left(\varphi^{n}\right)^{-1}\right)}{d s} \cdot \frac{d\left(p_{\tau}^{n+1} \circ\left(\varphi^{n}\right)^{-1}\right)}{d s} d s+\sum_{x \in \mathcal{V}_{h}^{0}} h_{i}^{n} \Psi(x) \cdot p_{c}^{n+1}(x)=0, \\
& \sum_{e \in \mathcal{E}_{h}^{n}} \int_{e} \frac{d^{2}\left(\Phi_{h}^{n+1} \circ\left(\varphi^{n}\right)^{-1}\right)}{d s^{2}} \cdot q_{\tau} \circ\left(\varphi^{n}\right)^{-1} d s+\int_{\Sigma} \frac{d\left(\Phi_{h}^{n+1} \circ\left(\varphi^{n}\right)^{-1}\right)}{d s} \cdot \frac{d\left(q_{\tau} \circ\left(\varphi^{n}\right)^{-1}\right)}{d s} d s=0 \\
& \sum_{x \in \mathcal{V}_{h}^{0}} h_{x}^{n}\left(\Phi^{n+1}(x)-\varphi^{n+1}(x)\right) \cdot q_{c}(x)=0 \\
& \varphi^{n+1}=\varphi^{n}+\Delta t u^{n+1},
\end{aligned}
$$

where $\mathcal{E}_{h}^{n}$ is the set of edges of the mesh $S_{h}^{n}$ of $\Sigma, \mathcal{V}_{h}^{n}$ its set of vertices and

$$
h_{x}^{n}=\frac{1}{2} \sum_{\substack{e \in \mathcal{E}_{h}^{0} \\ x \in \partial e}}\left|\varphi^{n}(e)\right|
$$




\subsection{A basic Algorithm}

The numerical algorithm we propose is the following one.

Algorithm 3.1. (1) Initialization of the initial velocity $u^{0}$ of the fluid and deformation $\varphi^{0}$ of the vesicles;

(2) Computation of the velocity $u^{n+1}$ and the deformations $\varphi^{n+1}$ at time $(n+1) \Delta t$ using (10-14);

(3) Remeshing. Rebuild a mesh $\mathcal{T}_{h}^{n+1}$ of the domain $\Omega$ that set of edges contains the edges of the mesh $\mathcal{S}_{h}^{n+1}=\varphi^{n+1}\left(\Sigma_{0}\right)$

(4) Go back to (2) until final time $T$ reached.

\subsection{Contacts}

The previous algorithm fails to take correctly into account possible contacts between the vesicles themselves or between the vesicles and the boundaries of the domain. It is known that rigid bodies could not get into contact in finite time in $2 \mathrm{~d}$ [12]. This suggests that is also impossible for deformable vesicles. Whatever the answer is, collisions always occur numerically. In order to prevent any mesh generation failure, we have to ensure that at each iteration the vesicles do not intersect themselves or the boundary of the domain. To this end, we perform an additional projection step of the deformation $\varphi^{n+1}$ on the set of deformations without (self-)intersection that does not cross the boundary of the domain. More precisely, instead of step (3) we compute the deformation $\varphi^{n+1} \in \mathcal{A}_{h, \varepsilon}$ such that

$$
d\left(\varphi^{n+1}, \widetilde{\varphi}^{n+1}\right)=\inf _{\varphi \in \mathcal{A}_{h, \varepsilon}} d\left(\varphi, \widetilde{\varphi}^{n+1}\right)
$$

where $\widetilde{\varphi}^{n+1}=\varphi^{n}+\Delta t u^{n+1} \circ \varphi^{n}, \mathcal{A}_{h, \varepsilon}$ is the set of admissible deformations and $d(\cdot, \cdot)$ is a distance function.

The distance function used is an approximation of the $H^{2}$ norm of $\varphi^{n+1}-\widetilde{\varphi}^{n+1}$ - the $H^{2}$ norm is not correctly defined as $\varphi^{n+1}$ and $\widetilde{\varphi}^{n+1}$ only belong to $H^{1}$, being P1-Lagrange elements. More precisely, for all elements $\varphi_{h}$ and $\psi_{h}$ in $V_{h}$, we set

$$
d\left(\phi_{h}, \psi_{h}\right)=\left(\left\|\phi_{h}-\psi_{h}\right\|_{L^{2}}^{2}+J_{h}\left(\phi_{h}-\psi_{h}\right)\right)^{1 / 2} .
$$

The set of admissible deformations is defined as

$$
\mathcal{A}_{h, \varepsilon}=\left\{\varphi_{h} \in V_{h} \text { such that } \operatorname{dist}(\varphi, \partial \Omega) \geq \varepsilon \text { and } \operatorname{dist}\left(\varphi_{h}(a), \varphi_{h}(b)\right) \geq \varepsilon \text { for all edges } a \text { and } b \text { of } \mathcal{S}_{h}, a \cap b=\emptyset\right\},
$$

$\varepsilon>0$ being a small security distance. The admissible set $\mathcal{A}_{h, \varepsilon}$ being not convex, problem (15) is not trivial. The minimizer of $d\left(\cdot, \widetilde{\varphi}^{n+1}\right)$ is computed using an internal approximation method introduced in [23] (see also [2] for a three dimensional application to aortic valves). It consists in solving a sequence of convex problems defined by

$$
d\left(\varphi_{p+1}^{n+1}, \widetilde{\varphi}^{n+1}\right)=\inf _{\varphi \in T\left(\varphi_{p}^{n+1}\right)} d\left(\varphi, \widetilde{\varphi}^{n+1}\right)
$$

with $\varphi_{0}^{n+1}=\varphi^{n}$, where $T(\cdot)$ is an application that maps all elements $\psi$ of $\mathcal{A}_{h, \varepsilon}$ to a convex neighborhood $T(\psi)$ of $\mathcal{A}_{h, \varepsilon}$ (see [23] for more details). For any $\psi \in \mathcal{A}_{h, \varepsilon}, T(\psi)$ is defined by

$$
T(\psi)=\left\{\varphi_{h} \in V_{h} \text { such that } F_{e, x}^{0}\left(\varphi_{h}\right) \leq 0 \text { and } F_{e, x}^{1}\left(\varphi_{h}\right) \leq 0 \text { for all edges } e \text { of } \mathcal{S}_{h}^{0}\right\},
$$

where for $i=0,1$,

$$
F_{e, x}^{i}\left(\varphi_{h}\right)=\varepsilon-n_{e, x}(\psi) \cdot\left(\varphi_{h}\left(e_{i}\right)-\varphi_{h}(x)\right),
$$

$e_{0}$ and $e_{1}$ being the extremities of the edge $e$ and $n_{e, x}(\psi)$ the unitary vector defined by

$$
\min _{x_{e} \in e} n_{e, x}(\psi) \cdot\left(\psi\left(x_{e}\right)-\psi(x)\right)=\operatorname{dist}(\psi(e), \psi(x)) .
$$

The dependence of the functions $F_{e, x}^{0}$ and $F_{e, x}^{1}$ with respect to $\psi$ in the definition of $T(\psi)$ is implicit and does not appear in the notations used. 


\subsection{Volume and Surface conservation}

Even for relatively short time simulations, the algorithm introduced in the previous section failed to correctly preserve both volume and perimeter of the blood cells. Indeed, it only preserves them up to an order one. To prevent large deviations of the volume or perimeter from their initial values, we modify the projection step over the set of deformations without intersection by adding the appropriate constraints to the minimization problem (15). Thus, at each iteration, the deformation $\varphi^{n+1}$ is computed solving

$$
d\left(\varphi^{n+1}, \widetilde{\varphi}^{n+1}\right)=\inf _{\varphi \in \mathcal{A}_{h, \varepsilon} \cap V_{h}^{a d}} d\left(\varphi, \widetilde{\varphi}^{n+1}\right)
$$

where $V_{h}^{a d}$ is the set of $P 1$ inextensional deformations with prescribed volume

$$
V_{h}^{a d}=\left\{\varphi \in V_{h} \text { such that } \ell_{e}(\varphi)=\ell_{e}\left(\varphi^{0}\right) \text { for all edges } e \text { of } \mathcal{S}_{h}^{0} \text { and } \operatorname{Vol}_{i}(\varphi)=\operatorname{Vol}_{i}\left(\varphi^{0}\right) \text { for all } i \in I\right\}
$$

where $I$ is the set of vesicles, $\operatorname{Vol}_{i}(\varphi)$ is the volume of the $i$-th vesicle and $\ell_{e}(\varphi)=|\varphi(e)|$ is the length of the deformed edge $\varphi(e)$. The volume and inextensibility constraints added in the minimization problem (16) are both not convex. To solve this problem, we use an iterative scheme, linearizing both constraints at each step. Thus, $\varphi^{n+1}$ is computed using the following algorithm.

Algorithm 3.2. (1) Initialization. Set $\varphi_{0}^{n+1}=\varphi^{n}$;

(2) For each $k>0$, compute $\varphi_{k}^{n+1}$ such that

$$
d\left(\varphi_{k}^{n+1}, \widetilde{\varphi}^{n+1}\right)=\inf _{\varphi \in \mathcal{A}_{h, \varepsilon} \cap V_{h, k}^{a d}} d\left(\varphi, \widetilde{\varphi}^{n+1}\right)
$$

where

$V_{h, k}^{a d}=\left\{\varphi \in V_{h}\right.$ such that $P_{e, k}(\varphi)=P_{e, k}\left(\varphi_{h}^{0}\right)$ for all edges e of $\mathcal{S}_{h}^{0}$ and $L_{i, k}(\varphi)=L_{i, k}\left(\varphi^{0}\right)$ for all $\left.i \in I\right\}$ with $P_{e, k}=D \ell_{e}\left(\varphi_{k-1}^{n+1}\right)$ and $L_{i, k}=D \operatorname{Vol}_{i}\left(\varphi_{k-1}^{n+1}\right)$;

(3) Stopping criterion. If $\left|\operatorname{Vol}_{i}\left(\varphi_{k}^{n+1}\right)-\operatorname{Vol}_{i}\left(\varphi_{k}^{0}\right)\right|<\delta_{V} \operatorname{Vol}_{i}\left(\varphi_{k}^{0}\right)$ and $\left\|\varphi_{k}^{n+1}(e)|-| \varphi_{k}^{0}(e)\right\|<\delta_{e}\left|\varphi_{k}^{0}(e)\right|$ for all edges e of $\mathcal{S}_{h}^{0}$ and all vesicles $i \in I$ then $S T O P$, else go back to (2).

\subsection{Complete Algorithm}

Algorithm 3.3. $\quad$ (1) Initialization of the initial velocity $u^{0}$ of the fluid and deformation $\varphi^{0}$ of the vesicles, set $n=0$;

(2) Computation of the velocity $u^{n+1}$ and deformations (before projection step) $\widetilde{\varphi}^{n+1}$ at time $(n+1) \Delta t$ solution of $(10-14)^{2}$;

(3) Projection step, computation of the deformation $\varphi^{n+1}$ at time $(n+1) \Delta t$

(a) Initialization. Set $\varphi_{0}^{n+1}=\varphi^{n}, \widetilde{\varphi}^{n+1}=\varphi^{n}+\Delta t u^{n+1}$ and $k=1$;

(b) Compute $\varphi_{k}^{n+1}$ such that

$$
d\left(\varphi_{k}^{n+1}, \widetilde{\varphi}^{n+1}\right)=\inf _{\varphi \in \mathcal{A}_{h, \varepsilon} \cap V_{h, k}^{a d}} d\left(\varphi, \widetilde{\varphi}^{n+1}\right) ;
$$

(i) Set $\varphi_{k, 0}^{n+1}=\varphi_{k-1}^{n+1}$ and $p=0$;

(ii) Compute $\varphi_{k, p+1}^{n+1}$ such that

$$
d\left(\varphi_{k, p+1}^{n+1}, \widetilde{\varphi}^{n+1}\right)=\inf _{\varphi \in T\left(\varphi_{k, p}^{n+1}\right) \cap V_{h, k}^{a d}} d\left(\varphi, \widetilde{\varphi}^{n+1}\right) ;
$$

${ }^{2} \varphi^{n+1}$ has to be replaced by $\widetilde{\varphi}^{n+1}$ in the set of equations (10-14) 
(iii) If

$$
d\left(\varphi_{k, p+1}^{n+1}, \varphi_{k-1, p+1}^{n+1}\right)<\delta / \Delta t
$$

set $\varphi_{k}^{n+1}=\varphi_{k, p+1}^{n+1}$ go to (3c), else increase $p$ and go back to $3(b) i i$;

(c) Stopping criterion. If

$$
\left|\operatorname{Vol}_{i}\left(\varphi_{k}^{n+1}\right)-\operatorname{Vol}_{i}\left(\varphi_{k}^{0}\right)\right|<\delta_{V} \operatorname{Vol}_{i}\left(\varphi_{k}^{0}\right) \text { and }\left\|\varphi_{k}^{n+1}(e)|-| \varphi_{k}^{0}(e)\right\|<\delta_{e}\left|\varphi_{k}^{0}(e)\right|
$$

for all edges e of $\mathcal{S}_{h}^{0}$ and all vesicles $i \in I$ then set $\varphi^{n+1}=\varphi_{k}^{n+1}$ and go to (4), else increase $k$ and go back to (3b);

(4) Remeshing. Rebuild a mesh $\mathcal{T}_{h}^{n+1}$ of the domain $\Omega$ that set of edges contains the edges of the mesh $\mathcal{S}_{h}^{n+1}=\varphi^{n+1}\left(\Sigma_{0}\right)$;

(5) Increase $n$ and go back to (2) until final time $T$ reached.

\section{Numerical Applications}

A typical human RBC, or erythrocyte is normally $8-10 \mu \mathrm{m}$ in diameter and $2 \mu \mathrm{m}$ in thickness. The RBCs' membrane has a bending elastic modulus of $k=1.8 \times 10^{-12}$ dyn-cm [9]. The RBCs are suspended in a fluid called blood plasma which is mostly composed of water ( $92 \%$ by volume). In the following simulations, we use the viscosity of water $\mu_{\text {out }}=1 \mathrm{mPa} \cdot \mathrm{s}$ as that of the exterior surrounding fluid.

\subsection{Case of a single RBC without external hydrodynamic forces}

Figure 1 illustrates a single RBC of $8 \mu \mathrm{m}$ in diameter in the equilibrium state (without any external hydrodynamic forces). As shown on the figure, a conformal mesh is used during the computation. The reduced volume has been chosen so that the equilibrium shape of the vesicle agrees with the observations [7].

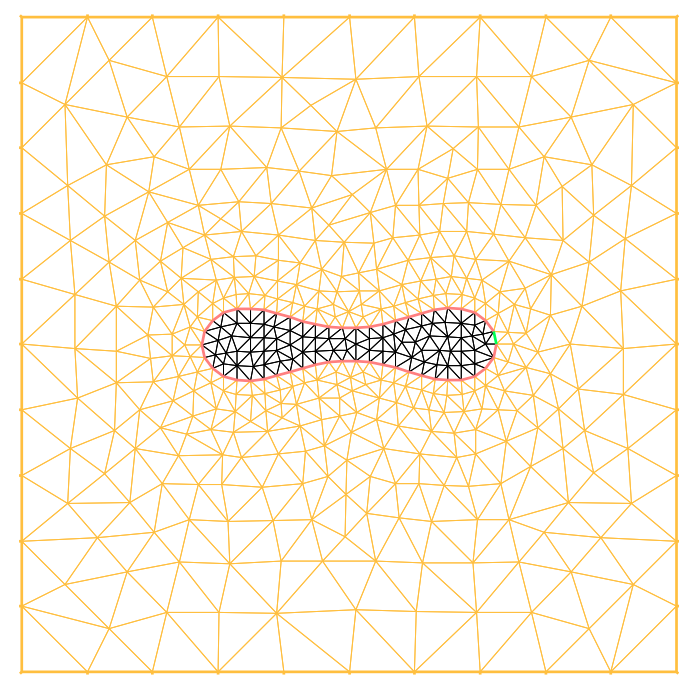

Figure 1. A single vesicle in equilibrium state. 


\subsection{In a shear flow}

In a shear flow, vesicles and RBCs show several dynamics depending on different parameters, such as the capillary number, the ratio between external and internal viscosity and confinement $[13,14,18]$. At least three different dynamics have been exhibited: tumbling, tank-treading and vacillating breathing. Figure 2 displays at different time steps the deformation of a $\mathrm{RBC}$ in a tank-treading motion. After a relaxation time, the shape of the $\mathrm{RBC}$ remains constant and the flow become stationary. Nevertheless, the deformation of the RBC is not constant as it rotates onto itself. An element of the membrane of the RBC has been marked with a small black disk, which enables us to visualize its motion. In the stationary state, the RBC forms a small constant angle with the vessel axis.

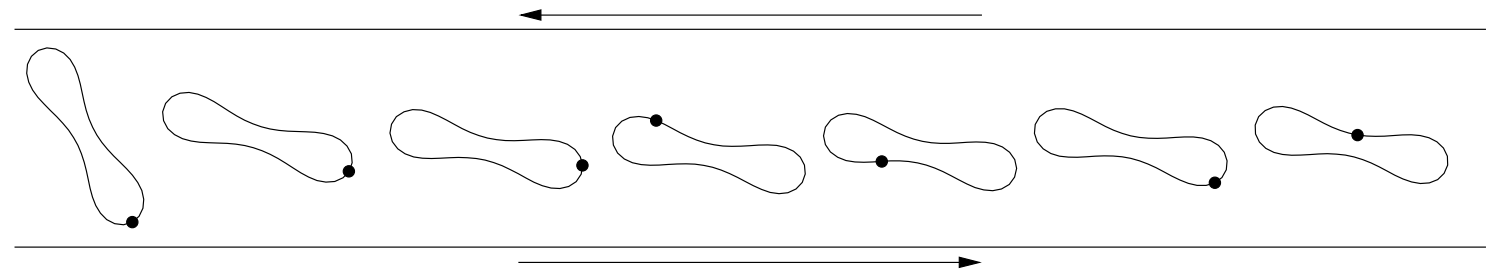

FiguRE 2. Stationary tank-treading motion of a single RBC under a shear flow with the shear rate of $2 \mathrm{~s}^{-1}$, at different time steps: $t=0 \mathrm{~s}, 2 \mathrm{~s}, 4 \mathrm{~s}, 30 \mathrm{~s}, 50 \mathrm{~s}, 70 \mathrm{~s}, 90 \mathrm{~s}$. Parameters used: diameter of the $\mathrm{RBC}=8 \mu \mathrm{m}, k=1.8 \times 10^{-12} \mathrm{dyn}-\mathrm{cm}, \mu_{\text {in }}=\mu_{\text {out }}=1 \mathrm{mPa} \cdot \mathrm{s}$.

When the ratio between the internal and external viscosities $\mu_{i n} / \mu_{\text {out }}$ increases, the dynamics of the RBC change to a tumbling motion (see Figure 3 ). The vesicle rotates periodically around its center. Note that, the rotation velocity is not constant. Close to the vertical position, the RBC is slightly deformed by the strong torque exerted by the flow and its rotation velocity reaches a maximum. This tumbling motion is combined with a tank-treading like dynamics when it approaches the horizontal state.

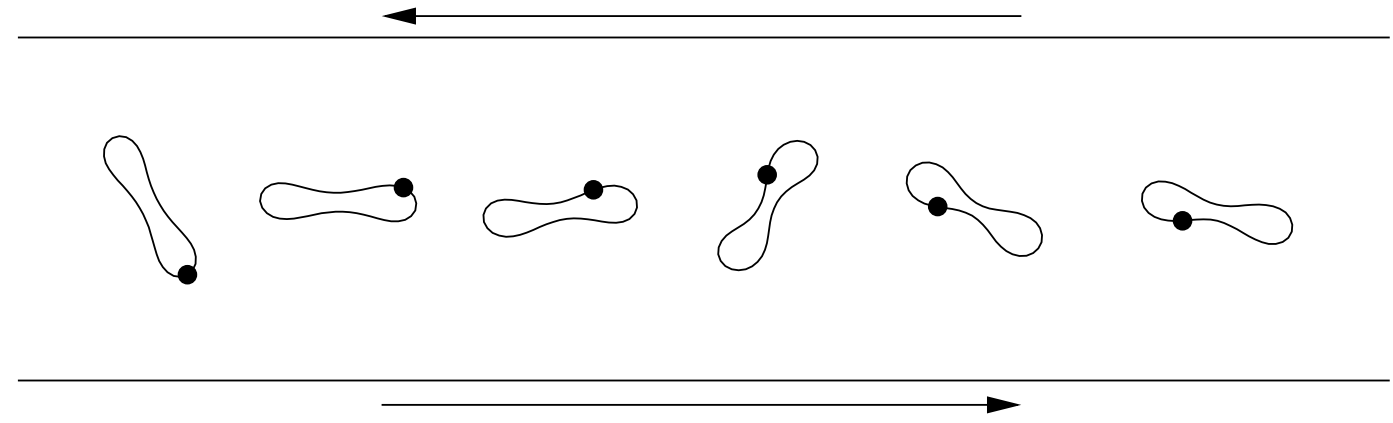

Figure 3. Tumbling motion of a single RBC under a shear flow with the shear rate of 10 $\mathrm{s}^{-1}$, at different time steps: $t=0 \mathrm{~s}, 2 \mathrm{~s}, 4 \mathrm{~s}, 5 \mathrm{~s}, 5.3 \mathrm{~s}, 5.5 \mathrm{~s}$. Parameters used: diameter of the $\mathrm{RBC}=10 \mu \mathrm{m}, k=1.8 \times 10^{-12}$ dyn-cm, $\mu_{\text {in }}=10 \mathrm{mPa} \cdot \mathrm{s}, \mu_{\text {out }}=1 \mathrm{mPa} \cdot \mathrm{s}$.

\subsection{Dynamics through capillary vessels}

The high deformability of RBCs enables them to go through vessels smaller than their diameter. To this end, they adopt a parachute shape as shown on Figure 4, where four RBCs are placed in a Poiseuille flow. Hydrodynamic forces prevent the RBCs from contacting the walls of the vessel. 

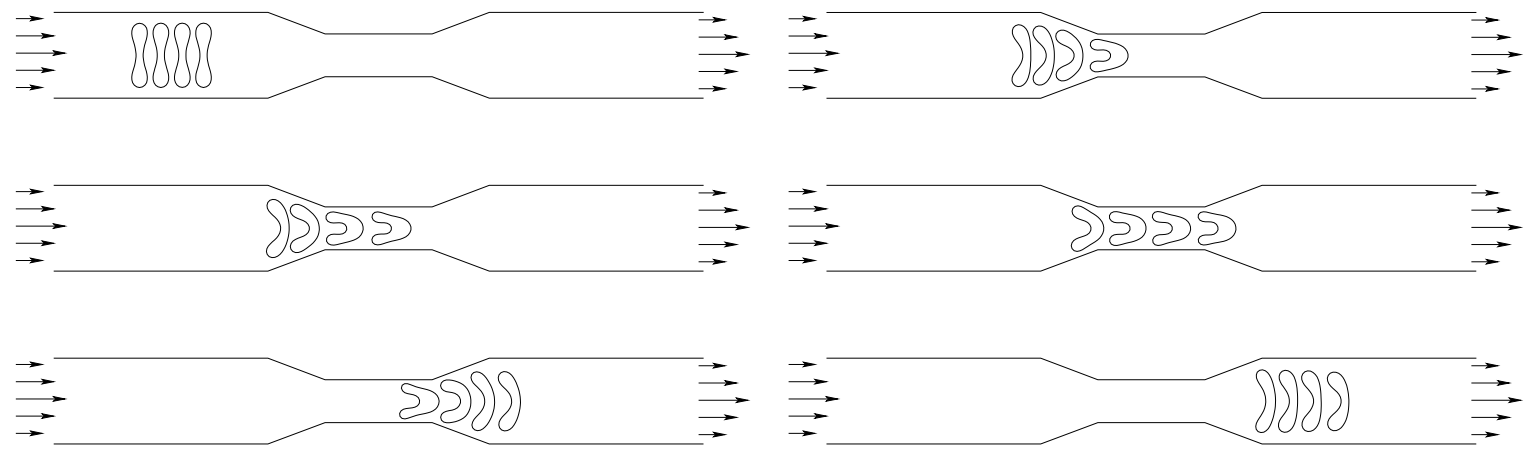

FiguRE 4. Simulation of 4 RBCs squeezed through a capillary vessel under a Poiseuille flow. From left to right, top to bottom: $t=0 \mathrm{~s}, 0.4 \mathrm{~s}, 0.5 \mathrm{~s}, 0.6 \mathrm{~s}, 0.8 \mathrm{~s}, 1.0 \mathrm{~s}$. Parameters used: diameter of the $\mathrm{RBCs}=8 \mu \mathrm{m}, k=1.8 \times 10^{-12}$ dyn-cm, $\mu_{i n}=\mu_{\text {out }}=1 \mathrm{mPa} \cdot \mathrm{s}$, velocity of the input flow at the center of vessel $=40 \mu \mathrm{m} / \mathrm{s}$.

\subsection{In a bifurcate vessel}

Figure 5 illustrates the initial configuration of four RBCs in a small vessel under a Poiseuille flow. They seem to get stuck when arriving the bifurcation where contacts between themselves and with the wall of the vessel are observed (Figure 6a). After a while, this state is overcome: the RBCs continue to go through the two vessel branches although contacts are still occurring (Figure 6b, 6c, 6d). Finally, each couple of RBCs has chosen a branch to pass along (Figure 6e, 6f). Due to the management of the contacts between the RBCs, the time of the computation is significatively increased for this simulation compared with the other cases presented here. The overall computation time is 4477 seconds of CPU time on a quad core $3 \mathrm{GHz} \mathrm{PC}$ compared to $139 \mathrm{~s}$ for the simulation of the tumbling motion of a single RBC displayed on Figure ??.

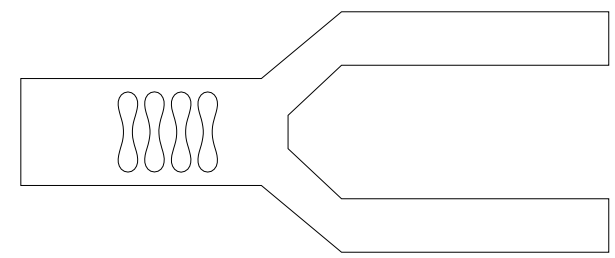

FIgURE 5. Four RBCs in a bifurcate vessel at initial time. Parameters used: diameter of the $\mathrm{RBC}=8 \mu \mathrm{m}, k=1.8 \times 10^{-12}$ dyn-cm, $\mu_{\text {in }}=\mu_{\text {out }}=1 \mathrm{mPa} \cdot \mathrm{s}$.

\section{Conclusion}

We have presented in this paper a method to simulate the motion of RBCs in the plasma flow in a bidimensional setting. Our algorithm enables to treat contacts between the RBCs themselves or contacts between the RBC and the walls. Moreover it preserves both the volume and the perimeter of the RBCs. Intersections between the RBCs' membrane or between the RBCs and the vessels' walls are forbidden by the introduction of an additional constraint that enforces a minimal distance between them. Several complex dynamics have been reproduced with our algorithm - tank-treading motion, tumbling motion and parachute shape - in good agreement with experimental observations. 

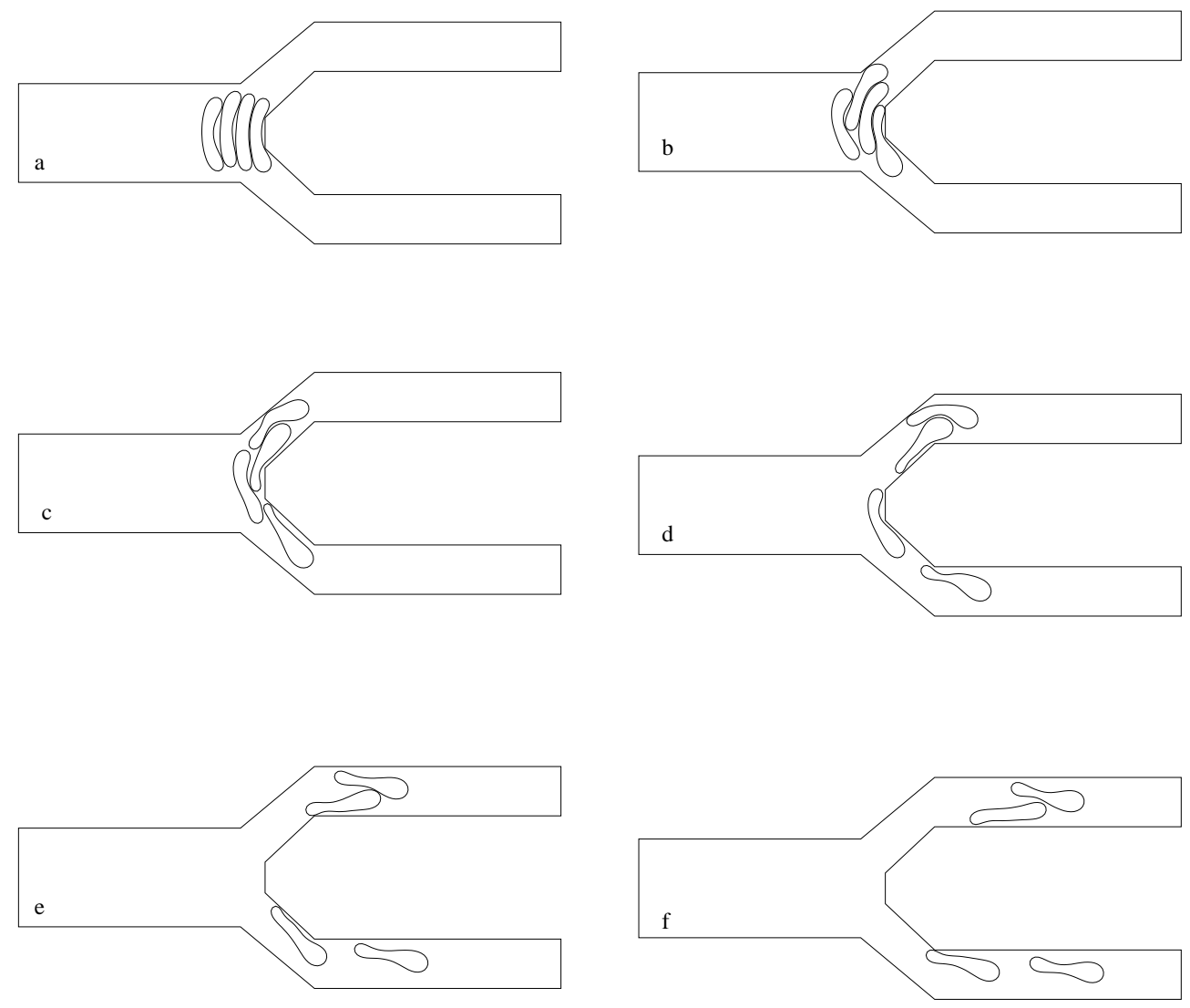

Figure 6. Simulation of 4 RBCs in a bifurcate vessel. From left to right, top to bottom: $t=0.32 \mathrm{~s}, 0.40 \mathrm{~s}, 0.52 \mathrm{~s}, 0.68 \mathrm{~s}, 0.84 \mathrm{~s}, 1.0 \mathrm{~s}$. Velocity of the input flow at the center of vessel $=40 \mu \mathrm{m} / \mathrm{s}$.

In the future, we intend to improve our algorithm so to be able to treat the case of a large number of vesicles in the same simulation. In the present version, the contact algorithm is quite time consuming for large numbers of vesicles (the number of constraints scales likes the square of the number of vesicles). The number of constraints taken into account could easly been reduced, as distant vesicles at a given time will not contact each other at the next time step. Nevertheless, it remains to be implemented. Furthermore, the three-dimensional case is still challenging, and is of major interest as it will allow to perform qualitative comparaison of the numerical results with experimental data (and not only qualitative comparaison).

This work was supported by the Marie Curie Research Training Network MRTN-CT-2004-505226 "Multi-scale modelling and characterisation for phase transformations in advanced materials" (MULTIMAT).

\section{REFERENCES}

[1] M. Abkarian and A. Viallat. Dynamics of vesicles in a wall-bounded shear flow. Biophysical Journal, 89(2):1055-1066, Aug 2005.

[2] M. Astorino, J.-F. Gerbeau, O. Pantz, and K.-F. Traoré. Fluid structure interaction and multi-body contact. application to aortic valves. Rapport de recherche INRIA, 6583, 2008.

[3] J. Beaucourt, F. Rioual, T. Séon, T. Biben, and C. Misbah. Steady to unsteady dynamics of a vesicle in a flow. Phys. Rev. E, 69(1):011906, Jan 2004. 
[4] T. Biben and C. Misbah. Tumbling of vesicles under shear flow within an advected-field approach. Phys. Rev. E, 67(3):031908, Mar 2003.

[5] N. Callens, C. Minetti, G. Coupier, M.-A. Mader, F. Dubois, C. Misbah, and T. Podgorski. Hydrodynamic lift of vesicles under shear flow in microgravity. EPL (Europhysics Letters), 83(2):24002 (6pp), 2008.

[6] V. Cristini and G. Kassab. Computer modeling of red blood cell rheology in the microcirculation: A brief overview. Annals of Biomedical Engineering, 33(12):1724-1727, Dec 2005

[7] W. Deuling, H.J.and Helfrich. Red blood cell shapes as explained on the basis of curvature elasticity. Biophysical Journal, 16(8):861-868, Aug 1976.

[8] Q. Du, C. Liu, and X. Wang. A phase field approach in the numerical study of the elastic bending energy for vesicle membranes. Journal of Computational Physics, 198(2):450 - 468, 2004.

[9] E. Evans. Bending elastic modulus of red blood cell membrane derived from buckling instability in micropipet aspiration tests. Biophys. J., 43(1):27 - 30, 1983.

[10] F. Hecht, O. Pironneau, A. Le Hyaric, and K. Ohtsuka. FreeFem++. Ecole Polytechnique.

[11] W. Helfrich. Elastic properties of lipid bilayers: Theory and possible experiments. Z. Naturforsch., 28c:693 - 703, 1973.

[12] M. Hillairet. Lack of collision between solid bodies in a 2D incompressible viscous flow. Comm. Partial Differential Equations, $32(7-9): 1345-1371,2007$

[13] V. Kantsler and V. Steinberg. Orientation and dynamics of a vesicle in tank-treading motion in shear flow. Phys. Rev. Lett., 95(25):258101, Dec 2005.

[14] V. Kantsler and V. Steinberg. Transition to tumbling and two regimes of tumbling motion of a vesicle in shear flow. Physical Review Letters, 96(3):036001, 2006.

[15] M. Kraus, W. Wintz, U. Seifert, and R. Lipowsky. Fluid vesicles in shear flow. Phys. Rev. Lett., 77(17):3685-3688, Oct 1996.

[16] Lefebvre, Aline. Modélisation numérique d'écoulements fluide/particules - prise en compte des forces de lubrification. PhD thesis, Université Paris Sud, Faculté des sciences d'Orsay, Orsay, France, 2007.

[17] Y. Liu and W. K. Liu. Rheology of red blood cell aggregation by computer simulation. Journal of Computational Physics, 220(1):139 - 154, 2006.

[18] M. Mader, V. Vitkova, M. Abkarian, A. Viallat, and T. Podgorski. Dynamics of viscous vesicles in shear flow. The European Physical Journal E: Soft Matter and Biological Physics, 19(4):389-397, Apr 2006.

[19] C. Misbah. Vacillating breathing and tumbling of vesicles under shear flow. Physical review letters, 96, 2006.

[20] L. Munn and M. Dupin. Blood cell interactions and segregation in flow. Annals of Biomedical Engineering, 36(4):534-544, 2008.

[21] H. Noguchi and G. Gompper. Fluid vesicles with viscous membranes in shear flow. Phys. Rev. Lett., 93(25):258102, Dec 2004.

[22] H. Noguchi and G. Gompper. Shape transitions of fluid vesicles and red blood cells in capillary flows. Proceedings of the National Academy of Sciences of the United States of America, 102(40):14159-14164, 2005.

[23] O. Pantz. A frictionless contact algorithm for deformable bodies. preprint CMAP, Ecole Polytechnique, R.I. $647,2008$.

[24] C. Pozrikidis. Numerical simulation of the flow-induced deformation of red blood cells. Annals of Biomedical Engineering, 31(10):1194-1205, 2003.

[25] C. Pozrikidis. Axisymmetric motion of a file of red blood cells through capillaries. Physics of Fluids, 17(3):031503, 2005.

[26] U. Seifert. Configurations of fluid membranes and vesicles. Advances in physics, 46:13 - 137, 1997.

[27] V. Vitkova, M. Mader, and T. Podgorski. Deformation of vesicles flowing through capillaries. EPL (Europhysics Letters), 68(3):398-404, 2004.

[28] J. Zhang, P. C. Johnson, and A. S. Popel. Red blood cell aggregation and dissociation in shear flows simulated by lattice boltzmann method. Journal of biomechanics, 41(1), 2008. 